tables enable one to avoid a great deal of the multiplication of the ordinates by the proper cosines, which is inherent in the calculation of the coefficients. Further tables include the conversion from polar to rectangular coordinates (useful in passing from the two Fourier coefficients corresponding to a particular frequency to its amplitude and phase), Darwin's scheme for a large number of ordinates $(p=121)$, tables of squares, square roots, $(\sin \alpha) / \alpha$, and other tables of interest in harmonic analysis. The last quarter of the book is devoted to problems and examples in harmonic analysis, illustrating the use of the various preceding tables; also to problems in the analysis of hidden periodicities.

The present volume should prove very useful to anyone engaged in frequent harmonic analysis.

\title{
H. PORITSKY
}

Tables of Partitions. By Hansraj Gupta. Madras, Indian Mathematical Society, 1939. $5+81$ pp.

This work contains two tables. The first table gives the number $P(n)$ of unrestricted partitions of $n$ for $n \leqq 600$. The second table is a table of double entry giving the number $(n, m)$ of partitions of $n$ the least element of which is $m$ for $n \leqq 300$. As is explained in the introduction, the second table was calculated by the recursion formula $(n, m)=(n-m, m)+(n+1, m+1)$ together with certain special properties of the symbol $(n, m)$; a specimen calculation is given. The first table is a result of the second since $P(n)=(n+1,1)$. Adequate checks have been applied to insure accuracy. The introduction also contains summaries of certain papers by G. N. Watson and D. H. Lehmer on partitions.

The Indian Mathematical Society and the University of the Punjab are to be commended for bearing the cost of publication of this work. The table of unrestricted partitions has previously been published in two parts in the Proceedings of the London Mathematical Society. This table has been used by Chowla to disprove one case of a result conjectured by Ramanujan. This work should be very valuable to anyone interested in the subject of partitions.

\section{T. A. Pierce}

Elementary Mathematics from an Advanced Standpoint. Geometry. By Felix Klein. Translated from the third German edition by E. R. Hedrick and C. A. Noble. New York, Macmillan, 1939. 9+214 pp., 141 figs.

Another volume of Klein's masterful lectures is available in Eng- 
lish! This book-it should be found on the shelves of every mathematical library-will be read with pleasure and profit alike by the scholar, the student, and the teacher. It preserves, even in translation, all those qualities for which the lectures of Klein are so justly celebrated.

The first volume of Klein's Elementarmathematik vom höheren Standpunkte aus was translated also by Professors Hedrick and Noble (reviewed in this Bulletin, vol. 39 (1933), pp. 495-496), and this, the second, is similar in character and purpose: "In the foreground I shall place, let me say, the encyclopedic ideal-you will be offered a survey of the entire field of geometry into which you can arrange, as into a rigid frame, all the separate items of knowledge which you have acquired in the course of your study, in order to have them at hand when occasion to use them arises. Only afterward shall I emphasize that interest in mathematical instruction ... " (see page 1). The emphasis is on ideas, as opposed to the details involved in their development, and on the interrelations between different fields.

Klein considers geometry from the point of view developed systematically in his Erlanger Programm of 1872 . His book has a wide appeal in spite of the fact that the last statement might seem to indicate a theoretical treatment of interest only to the mathematical specialist. The explanation is found in the large number of applications to a wide variety of problems.

The book is divided into three parts: Part I, "The Simplest Geometric Manifolds"; Part II, "Geometric Transformations"; Part III, "Systematic Discussion of Geometry and Its Foundations." In Part I we find a discussion of all the classical geometric manifolds, that is, classes of objects which are investigated in geometry. Many of these manifolds (various types of vectors, tensors, and so on) are of interest to physicists. A detail is an explanation of the theory of Amsler's polar planimeter. We find in Part II a discussion of affine transformations, projective transformations, higher point transformations, and transformations with a change of space element. It is shown that the axonometric mapping of space on a plane used in descriptive geometry is an affine transformation with a vanishing determinant. Relief perspective and geographical maps are considered. There is an application of contact transformations to the theory of $\operatorname{cog}$ wheels. In the first section of Part III we find a classification of geometries according to their groups and a discussion of invariant theory. The second section of Part III contains the material of immediate interest to prospective teachers of high school geometry. Two methods of developing plane geometry, different from that followed by Euclid, 
are outlined. One emphasizes motions; the other takes distance, angle, and congruence as the fundamental notions. Finally, there is a critical discussion of Euclid's Elements.

A reading of this book makes one aware of recent progress in mathematics. To Klein, writing in 1908, geometry was coordinate geometry. He makes only bare references to point sets as objects of geometric study and to Hilbert space. He gives only a brief discussion of homeomorphisms and even then only in euclidean 3-space. Geometric manifolds with the complete generality of our modern abstract spaces were then unknown. Metric spaces (in the sense of Hausdorff) had been introduced only in 1906, and topological spaces were not yet clearly defined. The types of geometry that now occupy the center of the stage were only beginning to emerge. Nevertheless, they too fit into the framework erected by Klein.

\section{G. B. PrICE}

Advanced Calculus. By Ivan S. Sokolnikoff. New York, McGrawHill, 1939. $10+446$ pp.

In most American colleges there is an abrupt discontinuity in mathematical education at the end of a first course in calculus. The drop in enrollment due to the completion of required mathematics by hordes of engineering students makes a sudden change in the needs and capabilities of the students. This discontinuity is perhaps more apparent to outsiders than to mathematicians themselves. It is the reason for the widely held popular belief that all useful mathematics ends with the calculus, that Newton made the last discoveries in mathematics, and that mathematics is dead except for a few frills indulged in by a handful of eccentrics. Even the name "Advanced Calculus" encourages this misconception-as though it were just calculus over again, with a few added tricks to be sure, but no new ideas.

It is in a second course in calculus that the number of students with primary interest in mathematics is for the first time an appreciable percentage of the total. The problem of the relative emphasis on method, that is, proofs, versus utility, to meet the respective needs of mathematics majors and students in the applied field, is as perplexing as any problem in the whole of mathematical education. Some sort of compromise is usually necessary. It would be very useful to the teacher if textbooks of good craftmanship were available with a variety of methods of effecting such a compromise. Unfortunately, a number of the existing books in this field are (or at least have the bulk of) treatises, and are not entirely suitable for textbook use. 